http://jmscr.igmpublication.org/home/ ISSN (e)-2347-176x ISSN (p) 2455-0450

crossref DOI: https://dx.doi.org/10.18535/jmscr/v9i7.06

Journal Of Medical Science And Clinical Research

IGM Publication

An Official Publication of IGM Publication

\title{
Comparison of two systems of classification of leprosy based on clinicopathological correlation
}

\author{
Authors \\ Dr Sapna Shrivastava ${ }^{1}$, Dr Chandra Mathur ${ }^{2}$ \\ ${ }^{1}$ PG Student, ${ }^{2}$ Professor \\ R.N.T Medical College, Udaipur, Rajasthan, India
}

\begin{abstract}
Aim and Objectives: To classify leprosy patients into multibacillary and paucibacillary types based on the number of body areas involved. To correlate histological diagnosis of skin biopsies of leprosy cases with clinical diagnosis using Ridley \& Jopling classification.

Material and Methods: The study group consisted of all clinical types of leprosy patients attending the dermatology OPD of MB Hospital, Udaipur during the study period 2011-2014. Complete dermatological examination was carried out in all the patients and the number of skin lesions were charted out. Body surface area was divided into 7 areas- Both upper limb 2 areas, both lower limbs 2 areas, anterior and posterior aspect of trunk 2 areas and head and neck 1 area. The number of involved area in each patient was counted and recorded. Each patient was given a score on a scale of 1-7 depending on the number of areas involved. Slit skin smear examination was performed in each patient. Skin biopsies were taken from the active lesions in all the leprosy patients and processed at department of pathology at RNT Medical College, Udaipur. They were stained by H\&E and modified fite stain and graded as per Ridley \& Jopling classification (1966) into IL, TT, BT, BB, BL \& LL.

Observation and Results: The present study was conducted on 100 untreated cases of leprosy, of these 8 were diagnosed as TT, 5 as BT, 36 as BB, 8 as BL,22 as LL and 21 as IL histologically. Clinical spectrum of leprosy cases in the present study revealed maximum cases $(60 \%)$ in borderline group $(B T+B B+B L)$, followed by $L L$ (21\%), TT (10\%) and least in IL group (9\%). In our study, out of 100 patients 59 showed positive SSS examination. $100 \%$ patients of LL \& BL groups showed positivity while 100\% patients of TT group showed negative results. In BB leprosy $72.2 \%$ patients were showed positive results. Only one patient (20\%) showed positive SSS examination in BT group. Most of the patients with negative results belonged to IL group (46.34\%). In the present study $41 \%$ of patients belonged to group with 1-5 skin lesions. $100 \%$ of clinically diagnosed TT, BT \& IL cases come under the category of 1-5 lesions, while clinicohistopathological concordance was seen in 8/10 (80\%) cases of TT and 5/20 (25\%) cases of BT. 59 patients presented with more than 5 skin lesions, 100\% clinically diagnosed cases of LL were confirmed histologically while one more case was detected on biopsy examination which was missed clinically. There were 14 cases of BB which presented with $>5$ lesions but histologically 27 cases were diagnosed. Only 7/24 cases were confirmed histologically with $>5$ lesions as $B L$.

Conclusion: A specific sub-typing of Leprosy can be done by histopathological examination. Histopathology provides confirmatory information for suspect cases which can be missed in clinical practice or epidemiological studies. Clinical diagnosis highly sensitive and specific in case of polar groups of leprosy. For the exact sub typing of borderline cases, clinical diagnosis holds a lower sensitivity and specificity. Our findings suggest that patients with involvement of 2 or less body areas can be classified as PB leprosy and those with more than 2 body areas involved can be classified as MB leprosy for the purposes of therapy. There is only a fair agreement between clinical and histopathological approaches.
\end{abstract}




\section{Introduction}

Leprosy or Hansen's disease was named after physician Gerhard Armauer Hansen. Leprosy also known as Hansen's disease is a chronic infectious disease that primarily affects the skin and peripheral nerves. ${ }^{[3]}$ It is a pleomorphic granulomatous disease with a spectrum of lesions ranging from paucibacillary epithelioid cell granulomas surrounded by abundant lymphocytes in tuberculoid forms of leprosy to lymphopenic lesions with bacilli laden foamy macrophages in lepromatous leprosy. Leprosy expresses itself in different clinicopathological forms depending on the immune status of the host. Between the two extremes of tuberculoid and lepromatous leprosy the disease evolves through borderline tuberculoid, borderline lepromatous and mid borderline (Ridley and Jopling,1966) ( WHO, 1966) ${ }^{[1]}$. In India and in most endemic countries for purpose of therapy, all leprosy patients are classified into paucibacillary (PB) and multibacillary (MB) types, on the basis of the number of skin lesions, with 5 lesions being the determining number. A count of body areas involved in leprosy is an alternative method of classifying leprosy for therapeutic purposes and is used in leprosy control programmes in Nepal. Body surface area is divided into 7 areas. Both upper limb 2 areas, both lower limbs 2 areas, anterior and posterior aspect of trunk 2 areas and head and neck 1 area. Various systems of classification namely WHO classification, the clinical classification and the histopathological classification are in use, for classification of leprosy patients. Ridley and Jopling were the first to suggest a subdivision of leprosy on an immunological basis into five types. ${ }^{[1]}$

\section{Material and Methods}

The study group consisted of leprosy patients attending the dermatology OPD of MB Hospital, Udaipur during the study period 2011-2014. The present study was conducted on skin biopsies of 100 clinically diagnosed untreated leprosy patients presenting to the department of dermatology STD and leprosy of MB Hospital, Udaipur. Skin biopsies were collected in OPD, preserved in formalin and processed in the Department of Pathology, RNT Medical College, Udaipur. The tissue sections were stained with haematoxylin and eosin stain and Z-N stain. Histological classification of the 100 cases was done and then was correlated with the clinical classification.

Patients with all clinical types of leprosy were included in the study. Complete dermatological examination was carried out in all the patients and the number of skin lesions were charted out. Body surface area was divided into 7 areas- Both upper limb 2 areas, both lower limbs 2 areas, anterior and posterior aspect of trunk 2 areas and head and neck 1 area. The number of involved area in each patient was counted and recorded. Each patient was given a score on a scale of 1-7 depending on the number of areas involved. Slit skin smear examination was performed in each patient. Skin biopsies were taken from the active lesions in all the leprosy patients and processed at department of pathology at RNT Medical College, Udaipur. They were stained by H\&E and modified fite stain and graded as per Ridley \& Jopling classification (1966) into I, TT, BT, BB, BL \& LL.

\section{Observation and Results}

The present study was conducted on 100 untreated cases of leprosy, of these 8 were diagnosed as TT, 5 as BT, 36 as BB, 8 as BL, 22 as LL and 21 as IL histologically. Clinical spectrum of leprosy cases in the present study revealed maximum cases $(60 \%)$ in borderline group (BT+BB+BL), followed by LL (21\%), TT (10\%) and least in IL group (9\%). In our study, out of 100 patients 59 showed positive SSS examination. $100 \%$ patients of LL \& BL groups showed positivity while $100 \%$ patients of TT group showed negative results. In BB leprosy $72.2 \%$ patients were showed positive results. Only one patient (20\%) showed positive SSS examination in BT group. Most of the patients with negative results belonged to IL group $(46.34 \%)$. 
In the present study one classification of patient is done on the basis of body area involved. 20\% patients presented with single body area involved. $50 \%$ patient of TT had single body area involvement while $100 \%$ patient of LL \& BL had more than one area involved. Maximum number of patients with single area involvement were seen in IL $(52.3 \%)$.

In the present study $47 \%$ of the patients presented with peripheral nerve thickening. $6 / 8(75 \%)$ of TT $\& 4 / 5(80 \%)$ of BT patients were found to have peripheral nerve thickening while it was seen in $24 / 36(66.67 \%)$ cases of BB. In LL group only one patient showed nerve thickening.

In our study, skin biopsy section of $73 \%$ of cases showed epidermal atrophy, out of these most of the cases were of $\mathrm{BB}(36 \%)$ and LL (22\%) subtypes. While $100 \%$ of the cases of LL, BL and BB leprosy showed epidermal atrophy, it was present in $60 \%$ of BT and $25 \%$ of TT cases.

Of all the cases, $65 \%$ showed the presence of a clear subepidermal zone or grenz zone. None of the biopsy of TT \& IL showed a grenz zone while $100 \%$ of LL showed the presence of grenz zone, while majority of $\mathrm{BT}(60 \%), \mathrm{BB}(91.6 \%)$ and $\mathrm{BL}$ $(87.5 \%)$ showed a grenz zone.

In the present study distribution of cases was studied according to type of granuloma, $13 \%$ of cases showed epithelioid granuloma formation, $66 \%$ showed histiocytic granuloma while absence of granuloma was noticed in $21 \%$ of the cases, all of which were of indeterminate type.

In the present study, highest bacterial index (5) was found in $59.9 \%$ of the LL cases and also in $12.5 \%$ of BL cases, while the lowest index (0) was observed in all cases of IL \& TT and $80 \%$ of BT cases. Bacterial index of 1 was found in $20 \%$ cases of BT. $83.3 \%$ of the cases of BB showed a bacterial index of 3 . bacterial index 4 was observed in $91.5 \%$ cases of BL and $40.1 \%$ cases of LL. Study of the bacterial indices forms an important stepping stone for the clinical categorisation of case as paucibacillary and multibacillary, henceforth helping in the management of the case. In our study Bacterial
Index was noted was noted in the range of 0-5. A complete absence of bacilli (B.I.-0) has been described for the diagnosis of TT (Jopling).

In the present study $41 \%$ of patients belonged to group with 1-5 skin lesions. $100 \%$ of clinically diagnosed TT, BT \& IL cases come under the category of 1-5 lesions, while clinicohistological concordance was seen in $8 / 10(80 \%)$ cases of TT and $5 / 20(25 \%)$ cases of BT. 59 patients presented with more than 5 skin lesions, $100 \%$ clinically diagnosed cases of LL were confirmed hitologically while one more case was detected on biopsy examination which was missed clinically. There were 14 cases of BB which presented with $>5$ lesions but histologically 27 cases were diagnosed. Only 7/24 cases were confirmed histologically with $>5$ lesions.

\section{Discussion}

Leprosy, a chronic disease caused by the acid-fast bacillus Mycobacterium leprae, presents a spectrum of clinical, bacteriological, immunological, and dermatopathological characteristics $^{[2]}$. In the 1960s, Ridley and Jopling proposed a histological classification scheme for leprosy that ranged in severity, beginning with early indeterminant (I) leprosy and continuing with polar tuberculoid (TT) leprosy, borderline tuberculoid (BT) leprosy, mid-borderline (BB) leprosy, borderline lepromatous (BL) leprosy, and polar lepromatous (LL) leprosy ${ }^{[1]}$. This scheme has the virtue of having a significant concordance between clinical, immunological, and pathological features, and its subdivisions correlate with the number of acid-fast bacilli present in the dermis, generally expressed on a logarithmic scale by the bacteriologic index (BI), for which scoring ranges from 0 to $<6$. Furthermore, the exact placement of individual patients in the spectrum of disease assists clinicians in determining the required intensity of antimicrobial therapy, the prospects for the development of reactional states, and whether a protective Th1 or Th2 response predominates. 


\section{JMSCR Vol||09||Issue ||07||Page 24-30||July}

Leprosy expresses itself in different clinicopathological forms depending on the immune status of the host ${ }^{[1,6]}$. Diagnosis of leprosy is based on different clinical parameters which involves detailed examination of skin lesions and peripheral nerves. Demonstration of acid-fast bacilli in slit skin smears by Ziehl- Neelsen's staining also aids in diagnosis of leprosy. A reliable diagnosis hinges around a good histopathological diagnosis and demonstration of bacilli in histopathological sections. Modified Fite's procedure has proved most valuable in demonstrating lepra bacilli in tissue sections. Clinical classification gives recognition only to gross appearances of the lesions, while the parameters used for the histopathological classification are well defined, precise and also take into account the immunological manifestations which enable it to successfully bridge the pitfalls in leprosy diagnosis. Histopathology provides confirmatory information for suspected cases which can be missed in clinical practice or epidemiological studies and helps in exact typing. Histology also gives indication of progression and regression of disease under treatment.
Table 1 shows comparison of no. of lesions with clinical \& histological diagnosis. In the present study $41 \%$ of patients belonged to group with 1-5 skin lesions. $100 \%$ of clinically diagnosed TT, BT \& IL cases come under the category of 1-5 lesions, while clinicohistopathological concordance was seen in $8 / 10(80 \%)$ cases of TT and 5/20 (25\%) cases of BT. 59 patients presented with more than 5 skin lesions, $100 \%$ clinically diagnosed cases of LL were confirmed histologically while one more case was detected on biopsy examination which was missed clinically. There were 14 cases of BB which presented with $>5$ lesions but histologically 27 cases were diagnosed. Only $7 / 24$ cases were confirmed histologically with $>5$ lesions as BL. In study by rao et al, in patients with single skin lesion, commonest type was BT leprosy (29 out of 34 patients). Histopathological features of BT leprosy observed in 13 out of 34 patients with 10 showing features of IL and 2 patients of BL leprosy. In the group of patients with 2-5 lesions, all 24 patients were classified clinically as BT, whereas on histopathology, only 16 out of 24 showed features of BT leprosy.

Table 1. Comparison of no. of lesions with SSS Exam in different subtypes of leprosy

\begin{tabular}{|c|c|c|c|c|c|c|c|c|c|c|c|c|c|c|c|}
\hline \multirow{3}{*}{$\begin{array}{l}\text { No. of } \\
\text { lesions }\end{array}$} & \multirow{3}{*}{$\begin{array}{c}\text { SSS } \\
\text { Exam } \\
\text { positive } \\
\end{array}$} & \multicolumn{12}{|c|}{ Different subtypes of leprosy } & \multirow{2}{*}{\multicolumn{2}{|c|}{ Total }} \\
\hline & & \multicolumn{2}{|c|}{$\mathrm{TT}$} & \multicolumn{2}{|l|}{$\mathrm{BT}$} & \multicolumn{2}{|l|}{$\mathrm{BB}$} & \multicolumn{2}{|l|}{$\mathrm{BL}$} & \multicolumn{2}{|l|}{ LL } & \multicolumn{2}{|l|}{ IL } & & \\
\hline & & Clin & HP & Clin & $\mathrm{HP}$ & Clin & HP & Clin & $\mathrm{HP}$ & Clin & $\mathrm{HP}$ & Clin & HP & cli & $\overline{\mathrm{HP}}$ \\
\hline $1-5$ & 2 & 10 & 8 & 20 & 5 & 1 & 9 & 1 & 1 & 0 & 0 & 9 & 18 & 41 & 41 \\
\hline$>5$ & 57 & 0 & 0 & 0 & 0 & 14 & 27 & 24 & 7 & 21 & 22 & 0 & 3 & 59 & 59 \\
\hline Total & 59 & 10 & 8 & 20 & 5 & 15 & 36 & 25 & 8 & 21 & 22 & 9 & 21 & 100 & 100 \\
\hline
\end{tabular}

Table 2 shows comparison of no. of body areas with clinical \& histological diagnosis- In the present study 60 patients had $>2$ body area involved. Maximum concordance was seen in LL group where 20/21 cases confirmed histologically with $>2$ body area involved. All the cases of TT \& IL presented with 1-2 body area involvement, while it was confirmed in $70 \%$ cases of TT histologically. In TT, 1 case was there where $>2$ body areas were involved. 18/20 cases of BT presented with 1-2 body area involvement while only 4 cases were confirmed histologically. In study by Rao et al, 42 out of 43 patients in whom one body area was involved were clinically diagnosed as TT,BT or IL type leprosy. 3 patients had clinical features of BT but had BL subtype on histopathology. Good clinicopathological relation was observed in patients with involvement of 4-7 body areas, with concordance in 33 out of 41 patients. 


\section{JMSCR Vol||09||Issue ||07||Page 24-30||July}

Table 2: Comparison of no. of body areas with SSS Exam in different subtypes of leprosy

\begin{tabular}{|c|c|c|c|c|c|c|c|c|c|c|c|c|c|c|c|c|}
\hline \multirow{2}{*}{$\begin{array}{l}\text { No. of } \\
\text { body } \\
\text { areas }\end{array}$} & \multirow{2}{*}{$\begin{array}{c}\text { No of } \\
\text { patients }\end{array}$} & \multirow{2}{*}{$\begin{array}{c}\text { SSS } \\
\text { positive } \\
\text { patients }\end{array}$} & \multicolumn{12}{|c|}{ Differant subtypes of leprosy } & \multirow{2}{*}{\multicolumn{2}{|c|}{ Total }} \\
\hline & & & \multicolumn{2}{|c|}{$\mathrm{TT}$} & \multicolumn{2}{|l|}{ BT } & \multicolumn{2}{|c|}{ BB } & \multicolumn{2}{|l|}{$\mathrm{BL}$} & \multicolumn{2}{|c|}{ LL } & \multicolumn{2}{|c|}{$\mathrm{IL}$} & & \\
\hline & & & $\begin{array}{c}\text { Cli } \\
\mathrm{n}\end{array}$ & HP & $\begin{array}{c}\text { Cli } \\
\mathrm{n}\end{array}$ & HP & $\begin{array}{c}\text { Cli } \\
\mathrm{n}\end{array}$ & HP & Clin & HP & Clin & HP & $\begin{array}{c}\mathrm{Cli} \\
\mathrm{n}\end{array}$ & HP & cli & HP \\
\hline $1-2$ & 40 & 3 & 10 & 7 & 18 & 4 & 1 & 9 & 2 & 0 & 0 & 2 & 9 & 18 & 40 & 40 \\
\hline$>2$ & 60 & 56 & 0 & 1 & 2 & 1 & 14 & 27 & 23 & 8 & 21 & 20 & 0 & 3 & 60 & 60 \\
\hline Total & 100 & 59 & 10 & 8 & 20 & 5 & 15 & 36 & 25 & 8 & 21 & 22 & 9 & 21 & 100 & 100 \\
\hline
\end{tabular}

Table 3 shows that In present study, 41 out of 100 belonged to patient with 1-5 lesions and 59 patients with $>5$ lesions. On basis of body areas involved, 40 patients were observed with 2 or $<2$ involvement and 60 had $>2$ areas involved.. There was a good correlation in the clinical classification (95\%) and histopathological typing (92.6\%) between these two groups. When clinical types
TT, BT and IL were compared between the two groups, $100 \%$ concordance was found in TT and IL leprosy and $90 \%$ concordance in BT leprosy. The majority of patients in 2 or $<2$ areas group belonged clinically $(92.5 \%)$ and histopathologically $(72.5 \%)$ to the TT, BT and IL groups (i.e. the paucibacillary forms of leprosy).

Table 3. Comparison of no. of body areas with no. of lesions in different subtypes of leprosy

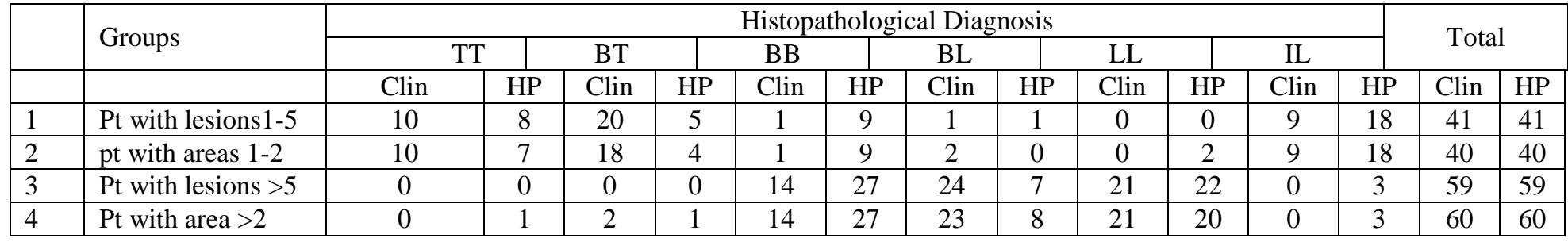

In study by Rao et al in 2005, 58 out of 108 patients belonged to the 5 or $<5$ lesions group and the rest 50 to that with $>5$ lesions. On basis of body area involved, 59 patients had 2 or $<2$ areas involvement and 49 had $>2$ body areas involved. There was good correlation in clinical classification (95\%) and histopathological typing (96\%) in study done by Rao et al. When clinical types TT, BT, IL were compared ,100\% concordance was found in TT and IL leprosy and 96\% concordance in BT leprosy. The majority of patients in 2 or $<2$ areas group belonged clinically
(95\%) and histopathologically (88\%) to the TT,BT and IL groups (i.e. the paucibacillary forms of leprosy)[9].

In our study when the group having more than 5 lesions was compared with the group having 3 or more body areas involvement, predominance of the multibacillary type of leprosy, both clinically $(100 \%$ and $96.6 \%$ respectively) and histopathologically (96\% and $95 \%$ respectively), was observed. Overall, the concordance between these two groups was $94 \%$ clinically and $96 \%$ histopathologically

Table 4. Comparison of clinical diagnosis with histopathological diagnosis

\begin{tabular}{|c|c|c|c|c|c|c|c|}
\hline \multirow{2}{*}{$\begin{array}{l}\text { CLINICAL } \\
\text { DIAGNOSIS }\end{array}$} & \multicolumn{6}{|c|}{ HISTOPATHOLOGICAL DIAGNOSIS } & \multirow{2}{*}{$\begin{array}{c}\text { PARITY } \\
(\%)\end{array}$} \\
\hline & TT & BT & BB & BL & LL & IL & \\
\hline TT (10) & 5 & 0 & 0 & 0 & 0 & 5 & $50 \%$ \\
\hline $\mathrm{BT}(20)$ & 3 & 4 & 9 & 0 & 0 & 4 & $20 \%$ \\
\hline $\mathrm{BB}(15)$ & 0 & 1 & 12 & 0 & 0 & 2 & $80 \%$ \\
\hline $\mathrm{BL}(25)$ & 0 & 0 & 13 & 5 & 7 & 0 & $20 \%$ \\
\hline $\operatorname{LL}(21)$ & 0 & 0 & 2 & 3 & 15 & 1 & $71.4 \%$ \\
\hline IL(9) & 0 & 0 & 0 & 0 & 0 & 9 & $100 \%$ \\
\hline 100 & 8 & 5 & 36 & 8 & 22 & 21 & $50 \%$ \\
\hline
\end{tabular}


In Rao et al study, when the group having $>5$ lesions was compared with the group having 3 or more body area involvement, predominance of multibacillary type of leprosy, both clinically (74 and $84 \%$ respectively) and histopathologically (65\% and $68 \%$ respectively) was observed. Overall, the concordance between groups was $94 \%$ clinically and $96 \%$ histopathologically ${ }^{[9]}$.

Maximum clinicohistopathological correlation was seen in IL followed by BB, LL, TT and minimum in BT \& BL. An overall parity of diagnosis was found in just $50 \%$ of cases. When cases of BT, BB and BL were clubbed together as one Borderline group between the two polar forms for clinical and histological diagnosis, high parity of $73.3 \%$ was observed. However, on considering TT and BT together as one group, and also BL and LL together as another group, clinicohistopathological concordance was $40 \%$ for the TT- BT group and $65.21 \%$ for the BL-LL group.

Using Kappa statistics it was found that, a substantial agreement was present only in LL, moderate agreement in TT, while a fair agreement was found in BT and BB. Only slight agreement was found in BL. Sensitivity and specificity of clinical examination was compared against histopathological examination and it was found that excluding IL cases, high degree of sensitivity and specificity was found in cases of LL followed by TT. Clinical diagnosis was least sensitive in BB All the cases diagnosed as IL clinically were confirmed to be the same histologically making the clinical diagnosis $100 \%$ specific in IL cases (Table 5).

Table 5. Comparison of the two diagnostic methods using kappa statistics

\begin{tabular}{|lcccccc|}
$\begin{array}{l}\text { CLINICAL } \\
\text { DIAGNOSIS }\end{array}$ & Sensitivity & Specificity & PPV & NPV & Kappa Statistics & Inference \\
\hline TT(10) & $62.5 \%$ & $94.56 \%$ & $50 \%$ & $98.84 \%$ & 0.51 & Moderate agreement \\
\hline BT(20) & $80 \%$ & $83 \%$ & $20 \%$ & $98.7 \%$ & 0.26 & Fair agreement \\
\hline BB(15) & $33.3 \%$ & $95.3 \%$ & $80 \%$ & $71.76 \%$ & 0.33 & Fair agreement \\
\hline BL(25) & $62.5 \%$ & $78.3 \%$ & $20 \%$ & $96.0 \%$ & 0.20 & Slight Agreement \\
\hline LL(21) & $68.18 \%$ & $92.3 \%$ & $71.4 \%$ & $91.1 \%$ & 0.61 & Substantial Agreement \\
\hline IL(9) & $42.85 \%$ & $100 \%$ & $100 \%$ & $63.63 \%$ & 0.54 & Moderate Agreement \\
\hline Total(100) & $58.22 \%$ & $90.57 \%$ & $56.9 \%$ & $86.61 \%$ & 0.40 & Fair agreement \\
\hline
\end{tabular}

\section{Conclusion}

A specific sub-typing of Leprosy can be done by histopathological examination. Histopathology provides confirmatory information for suspect cases which can be missed in clinical practice or epidemiological studies. Clinical diagnosis is highly sensitive and specific in case of polar groups of Leprosy. For the exact subtyping of borderline cases, clinical diagnosis holds a lower sensitivity and specificity .Our findings suggest that patients with involvement of 2 or less body areas can be classified as PB leprosy and those with more than 2 body areas involved can be classified as MB leprosy for the purposes of therapy. There is only a fair agreement between clinical and histopathological approaches.

\section{References}

1. Ridley D S \& Jopling W H. Classification of leprosy according to immunity: a fivegroup system. Int. J. Leprosy 34:255-73, 1966.

2. Elder DE, Elenitsas R, Jaworsky C, Johnson B. Lever's histopathology of the skin. $10^{\text {th }}$ ed. Philadelphia: Lippincott Williams and Wilkins; 2009. p. 569-77.

3. Park. K. Epidemiology of communicable diseases. Park's textbook of preventive \& social medicine. $20^{\text {th }}$ ed. Jabalpur(India): M/s Banarasidas Bhanot Publishers; 2009. p. 264-78. 
4. Ananthanarayan R. Panikar CKJ. Mycobacterium- III: Mycobacterium leprae: Ananthanarayn and Paniker's text book of microbiology. $8^{\text {th }}$ ed. Chennai: Orient Longman Pvt Ltd; 2009. p. 370-6.

5. Thangaraj RH, Hawalkar SJ. Diagnosis in leprosy. In: Leprosy for medical practitioners and para medical workers, Ciba Giegy; 2002. p. 39-41.

6. Jopling W.H. McDougall AC. The disease. In: Hand book of Leprosy. $5^{\text {th }}$ ed. New Delhi: CBS publishers and distributors; 2005. p. 10-49.

7. Abbas AK. Diseases of immunity. In: Kumar V, Abbas AK, Fausto N, editors.Robbins and Cotran pathologic basis of disease. $7^{\text {th }}$ ed. Philadelhpia: Saunders; 2004. p. 193-267.

8. Dubey GK, Joglekar VK, Grover S, Chaubey BS. Correlation of clinical and histopathological classification of leprosy. Lepr Ind 1981;53(4):562-5.

9. Rao P, Sujai S, Srinivas D, Lakshmi T: Comparison of two systems of classification of leprosy based on number of skin lesions and number of body areas involved- A clinicopathological concordance study. Indian J Dermatology Venerology Leprology 2005; 71:14-9. 